\title{
A Stopped-Flow/Rapid-Quenching Study of the Reaction of 1,1-Diphenylethylene and Sulfuric Acid*
}

\author{
Kunihide TAKARABE and Toyoki KUNITAKE** \\ Department of Organic Synthesis, Faculty of Engineering, Kyushu University, \\ Hakozaki, Higashi-ku, Fukuoka 812, Japan.
}

(Received September 29, 1979)

\begin{abstract}
The reaction of 1,1-diphenylethylene with sulfuric acid was studied at $30^{\circ} \mathrm{C}$ in dichloroethane by stopped-flow/rapid-scan spectroscopy coupled with the rapid-quenching technique. Rapid-scan spectra showed the formation and decay of two species absorbing at 435 and $465 \mathrm{~nm}$ during a period of less than one second. These species were detected only when sulfuric acid was $100 \%$ or more. The $435-\mathrm{nm}$ species was the monomeric cation formed by protonation, and the 465-nm species was conceivably a charge-transfer complex of diphenylethylene and $\mathrm{SO}_{3}$. The latter species eventually gave cyclic sulfones.

KEY WORDS Diphenylethylene / Sulfuric Acid / Cationic Polymerization / Stopped-Flow Technique / Rapid-Scan Spectroscopy /
\end{abstract}

In a previous article, we described the stoppedflow/rapid-quenching study of the reaction of 1,1diphenylethylene (DPE) and $\mathrm{CF}_{3} \mathrm{SO}_{3} \mathrm{H}^{1}{ }^{1}$ The reaction was fairly straightforward and the very fast protonation was followed by the slower formation of the cyclic dimer:

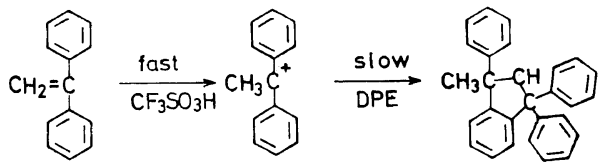

In contrast, the reaction of diphenylethylene with other cationic initiators appears much more complex. For example, Masure et al. ${ }^{2}$ observed three successive stages of initiation (formation of the carbocation) in the reaction with $\mathrm{AlCl}_{3}$. Having completed the stopped-flow/rapid-quenching study of the $\mathrm{CF}_{3} \mathrm{SO}_{3} \mathrm{H}$-catalyzed dimerization, we next chose to examine the reaction of diphenylethylene with sulfuric acid. The $\mathrm{H}_{2} \mathrm{SO}_{4}$-initiation of vinyl monomers has been extensively studied, and the present study is expected to help elucidate the initiation mechanism by sulfuric acid. It also appeared advisable to study this system prior to working on the more complex system initiated by

\footnotetext{
* Contribution No. 551 from Department of Organic Synthesis.

** Correspondence should be addressed to this author.
}

Friedal-Crafts catalysts.

\section{EXPERIMENTAL}

\section{Materials}

The purifications of diphenylethylene and dichloroethane are described in the accompanying paper. ${ }^{1}$ The " $100 \%$ " sulfuric acid was prepared by mixing calculated amounts of commercial concentrated sulfuric acid $(95 \%)$ and $30 \%$ fuming sulfuric acid. ${ }^{3}$ Sulfuric acids other than $100 \%$ were prepared similarly. These mixtures were added to dry dichloroethane and allowed to stand at room temperature for 2-3 days or one week and the organic supernatant was used as the initiator solution. The concentration of the $\mathrm{H}_{2} \mathrm{SO}_{4}$ moiety in dichloroethane was determined by titration with thymol blue as indicator. The original initiator solution was diluted with dry dichloroethane when necessary.

\section{Reaction Procedure}

The reaction was followed by a stoppedflow/rapid-scan spectrophotometer (Union Giken Co., Model RA 1300). The experimental procedure was described before. ${ }^{4}$

Diphenylethylene- $\mathrm{H}_{2} \mathrm{SO}_{4}$ Adduct

Under conditions of vigorous shaking, ten $\mathrm{ml}$ of a 
dichloroethane solution of diphenylethylene were injected to $50 \mathrm{ml}$ of a dichloroethane solution of $106 \%$ sulfuric acid in $1-2 \mathrm{~s}$ under dry nitrogen. The final concentrations were $56 \mathrm{mM}$ for diphenylethylene and $24 \mathrm{mM}$ for sulfuric acid. The reaction was quenched after $20 \mathrm{~min}$ by adding $20 \mathrm{ml}$ of $0.5 \mathrm{M}$ $\mathrm{CH}_{3} \mathrm{ONa}$ in $\mathrm{CH}_{3} \mathrm{OH}$. The resulting mixture was washed with water, dried over $\mathrm{CaCl}_{2}$ and the solvent was stripped off. The residue was recrystallyzed from methanol to give colorless plates; $\mathrm{mp} 154-155^{\circ} \mathrm{C}$, yield $34 \%$.

\section{RESULTS AND DISCUSSION}

\section{Rapid-Scan Spectra and Influence of Percentage of Sulfuric Acid on the Reaction}

When $8.8 \times 10^{-5} \mathrm{M}$ of diphenylethylene was mixed with excess sulfuric acid $(15.6 \mathrm{mM})$ in dry dichloroethane $\left(\left[\mathrm{H}_{2} \mathrm{O}\right]=2.1 \mathrm{mM}\right)$, complex rapidscan spectra shown in Figure 1 could be observed. The spectra were composed of two independent peaks with $\lambda_{\max }$ at 435 and $465 \mathrm{~nm}$. The 465 -nm peak appeared at an early stage of the reaction (e.g., $20 \mathrm{~ms}$ ). Two peaks could be seen within $40-60 \mathrm{~ms}$ of the reaction period, but these spectra consisted mainly of the $435-\mathrm{nm}$ peak at $100-300 \mathrm{~ms}$. A new peak at $600 \mathrm{~nm}$ appeared at a later stage. The species with $\lambda_{\max }=465 \mathrm{~nm}$ was more sensitive to the water concentration than the other species, and its formation depended very much on the preparation of the sulfuric acid. Therefore, sulfuric acid of different percentages was intentionally prepared and the effectiveness of the sulfuric acids as initiator was examined.

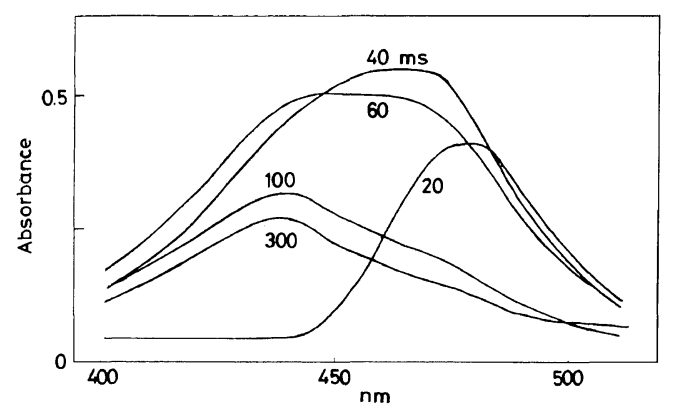

Figure 1. Rapid scan spectra at different reaction times: $30^{\circ} \mathrm{C}$; solvent, dichloroethane; DPE, $8.8 \times 10^{-5} \mathrm{M} ; 106 \%$ sulfuric acid, $16 \mathrm{mM} ; \mathrm{H}_{2} \mathrm{O}, 2.1 \mathrm{mM}$.
95\% and $98 \%$ Sulfuric Acids as Initiator. The sulfuric acid in these percentages was not sufficiently soluble in dichloroethane (at most $2 \mathrm{mM}$ ) and were ineffective as initiator. The peaks at 435 and $465 \mathrm{~nm}$ were very small in rapid-scan spectra or did not appear at all. These sulfuric acids are commercial $95 \%$ sulfuric acid itself and a mixture of the necessary amounts of $95 \%$ sulfuric acid and fuming $(30 \%$ excess) sulfuric acid. However, the exact percentage of sulfuric acid in dichloroethane is not known since the percentage of $\mathrm{H}_{2} \mathrm{SO}_{4}$ may change during its dissolution in dry dichloroethane due to the following equilibrium.

$$
\mathrm{H}_{2} \mathrm{SO}_{4} \rightleftarrows \mathrm{SO}_{3}+\mathrm{H}_{2} \mathrm{O}
$$

Nevertheless, the present observation suggests that extra water readily suppress the fast reaction of sulfuric acid and diphenylethylene.

$100 \%$ Sulfuric Acid as Initiator. The effectiveness of $100 \%$ sulfuric acid as an initiator was found to depend on the period of dissolution of the sulfuric acid in dry dichloroethane. In one series of experiments, $100 \%$ sulfuric acid with a dissolution period of 2-3 days was used as the initiator:

$$
\left[\mathrm{H}_{2} \mathrm{SO}_{4}\right]=5.4 \times 10^{-3} \mathrm{M},[\mathrm{DPE}]=4.4 \times 10^{-4} \mathrm{M} \text {. }
$$

Only the $465-\mathrm{nm}$ peak appeared immediately after mixing $(20-40 \mathrm{~ms})$, but the $435-\mathrm{nm}$ peak became predominant $150-200 \mathrm{~ms}$ later. When $1 \mathrm{mM}$ of $\mathrm{H}_{2} \mathrm{O}$ was added to the monomer solution, only the 435$\mathrm{nm}$ peak was seen with less absorbance. On the other hand, when $100 \%$ sulfuric acid with a dissolution period of one week was used $\left(\left[\mathrm{H}_{2} \mathrm{SO}_{4}\right]=1.94 \times 10^{-2} \mathrm{M},[\mathrm{DPE}]=1.21 \times 10^{-4} \mathrm{M}\right)$, only the $435-\mathrm{nm}$ peak appeared and its intensity reached a maximum in $100-150 \mathrm{~ms}$.

$106 \%$ Sulfuric Acid as Initiator. Similar to the above experiments, the two types of $106 \%$ sulfuric acid in dichloroethane were prepared with different dissolution periods. When the dissolution period was 2-3 days, the rapid-scan spectra contained only one peak at $465 \mathrm{~nm}$ which reached its maximal values at $50 \mathrm{~ms}$ :

$$
\begin{gathered}
{\left[\mathrm{H}_{2} \mathrm{SO}_{4}\right]=(7.95-11.0) \times 10^{-3} \mathrm{M},} \\
{[\mathrm{DPE}]=(2.15-51.8) \times 10^{-5} \mathrm{M} .}
\end{gathered}
$$

When the dissolution period was one week, the 465 $\mathrm{nm}$ peak was broader and the 435 -nm peak could be seen as the shoulder; see Figure 1. The latter peak became more apparent at a later stage of the reaction. 


\section{Determination of Reaction Products}

According to the preceding results, the formation of the unstable intermediates depends very much on the preparation of the dichloroethane solution of sulfuric acid. Two peaks were observed at 435 and $465 \mathrm{~nm}$ in the rapid-scan spectrum. The 435 -nm peak is assigned to the monomer cation as discussed in detail in a preceding paper. ${ }^{1}$ On the other hand, the 465-nm peak was observed only when the percentage of sulfuric acid was 100 or more, and this peak was shorter-lived and more sensitive to water than the monomer cation. Thus, we attempted to characterize this species by a rapid-quenching experiment.

The initiator used was $106 \%$ sulfuric acid with a dissolution period of $2-3$ days, since it produced only a 465-nm peak. After a given reaction period, the reaction mixture was quenched, washed with water, and subjected to liquid chromatography. Three major peaks appeared, depending on the reaction condition. The monomer peak appeared at a retention time of $11 \mathrm{~min}$ under standard chromatographic conditions, when the reaction period was short $(<1 \mathrm{~s})$. With longer reaction periods, the monomer peak disappeared completely, as seen from the liquid chromatogram of the quenched mixture in Figure 2. The absence of the methoxy-terminated product of the monomer cation 1 was confirmed by NMR spectroscopy.

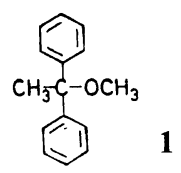

This is consistent with the fact that the 435-nm peak was not observed in the rapid-scan spectrum under these particular reaction conditions. Broad peaks appearing at 5-7 $\mathrm{min}$ in liquid chromatography are attributed to unidentified reaction products formed in the absence of monomer.* The two products eluting at $8 \mathrm{~min} 30 \mathrm{~s}$ and $9 \mathrm{~min}$ should thus be associated with the $465-\mathrm{nm}$ peak. It must be noted that these products were obtained

* Fifty $\mathrm{ml}$ of a dichloromethane solution of $98 \%$ sulfuric acid $(80 \mathrm{mM})$ was thoroughly mixed with $20 \mathrm{ml}$ of $1 \mathrm{M}$ $\mathrm{CH}_{3} \mathrm{ONa}$ in methanol, washed with water and dried. The resulting mixture gave rise to several broad peaks at 5-10 min under the standard condition of liquid chromatography. Its NMR spectrum possessed triplets at 3.7 and $4.5 \mathrm{ppm}$ and complex peaks at $0-2 \mathrm{ppm}$.

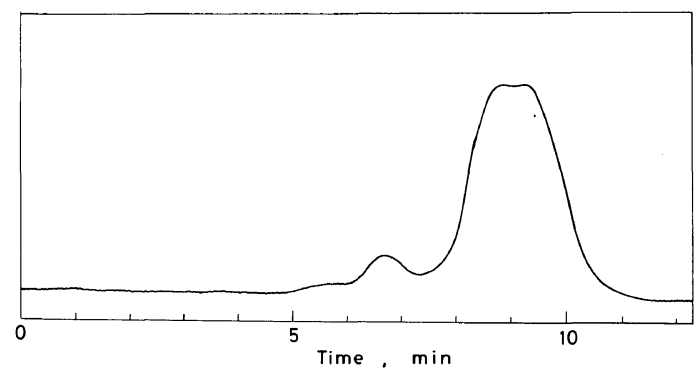

Figure 2. Liquid chromatogram of the quenched mixture. Reaction condition: temp, $30^{\circ} \mathrm{C}$; solvent, dichloroethane; DPE, $0.58 \mathrm{mM}$; $106 \%$ sulfuric acid, $27 \mathrm{mM}$; quenched by $60 \mathrm{mM} \mathrm{CH} \mathrm{CH}_{3} \mathrm{ONa}$ in $\mathrm{CH}_{3} \mathrm{OH}$. Chromatographic condition: instrument, Hitachi HLC 635; Hitachi gel 3019; $8 \phi \times 500$; methanol/

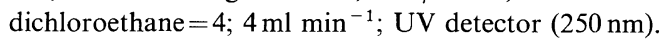

irrespective of whether quenching was conducted during the time scale of rapid-scan spectroscopy (i.e., the reaction period of $50-200 \mathrm{~ms}$ ) or the quenching time was much longer $(10-60 \mathrm{~min})$ than the lifetime of the 465-nm peak. This indicates that the product was not derived directly from the quenching of the unstable intermediate.

When the initial ratio of $\mathrm{H}_{2} \mathrm{SO}_{4}$ to the monomer was less than 10 , the 8.5 -min peak was predominant. This fraction was collected after recycling, removal of solvent in vacuo and recrystallization of the resulting solid from methanol to give colorless plates, $\mathrm{mp} 154-155^{\circ} \mathrm{C}$. The same compound can be synthesized on a larger scale as described in the EXPERIMENTAL. The analytical data of this compound are as follows: mass spectrum $(70 \mathrm{eV}) \mathrm{m} / \mathrm{e}$ $241\left(\mathrm{P}^{+}\right)$; IR (KBr) $3060\left(v_{\mathrm{C}-\mathrm{H}}\right), 1265,1145 \mathrm{~cm}^{-1}$ $\left(v_{\mathrm{SO}_{2}}\right)$; molecular weight (vapor pressure osmometry), 234. Anal. Calcd for $\mathrm{C}_{14} \mathrm{H}_{10} \mathrm{SO}_{2}$ : C, $69.40 \% ; \mathrm{H}$, $4.17 \%$. Found: C, $69.25 \%$; H, $4.12 \%$. An ${ }^{1} \mathrm{H}$ NMR spectrum of this compound is shown in Figure 3a. There is a multiplet peak at 7.4-7.9 ppm (9H) attributable to the aromatic protons and a singlet at $6.6 \mathrm{ppm}(1 \mathrm{H})$. These data are consistent with 3phenylbenzo[2,3-b]thiophene-1,1-dioxide:

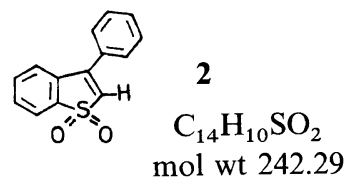




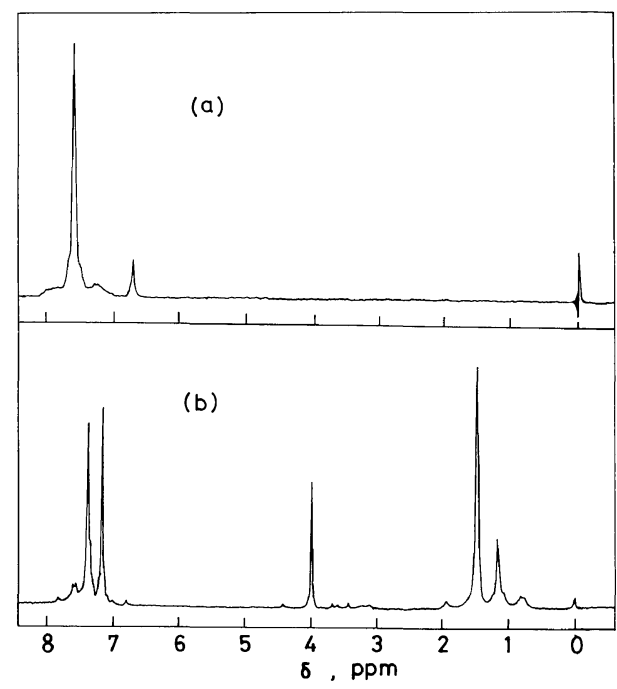

Figure 3. ${ }^{1} \mathrm{H}$ NMR spectra of the quenched product in $\mathrm{CDCl}_{3}$ : a, component eluting at $8 \mathrm{~min} 30 \mathrm{~s}$; b, run 5 in Table I.

When the $\left[\mathrm{H}_{2} \mathrm{SO}_{4}\right] /[\mathrm{DPE}]$ ratio was larger than 10 , the 9-min peak was commonly observed in liquid chromatograms. This fraction was separated and the solvent removed to leave a trace amount of white solid. An NMR spectrum was obtained without further purification. The spectrum is rather complex as shown in Figure 3b, since the sample contains unidentified quenching products formed in the absence of the monomer. The NMR peaks of Figure $3 \mathrm{~b}$ at $0-2 \mathrm{ppm}$ are very similar to those of the sample obtained in the absence of monomer. The sharp singlet $(6.6 \mathrm{ppm})$ observed in Figure $3 \mathrm{a}$ is lacking. Instead, a sharp singlet is found at $4.0 \mathrm{ppm}$. This is assigned to the methylene proton adjacent to phenyl sulfone.* The relative area of this peak and the pehnyl proton peak $(7.0-7.5 \mathrm{ppm}$, the chloroform peak at $7.25 \mathrm{ppm}$ excluded) is approximately $1: 4.5$. These data strongly suggest that the products have the structure of $\mathbf{3}$. In this structure, $X$ is probably the sulfate moiety, though this could not be confirmed.

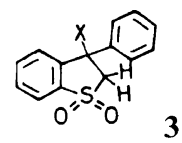

* $\delta=4.3 \mathrm{ppm}$ is given for the $\alpha$-methylene proton of $p$ hexadecylsulfonylaniline ${ }^{5}$
Table I summarizes the relation of the product ratio ( $2 v s .3)$ with the reaction condition. Under the condition of $\left[\mathrm{H}_{2} \mathrm{SO}_{4}\right] /[\mathrm{DPE}] \geq 10$ (entry $1-3$ ), the ratio of 3 increased with the reaction time. The formation of 3 is favored when $\left[\mathrm{H}_{2} \mathrm{SO}_{4}\right] /[\mathrm{DPE}]$ is large (compare entry 2,4 , and 6 ). These data suggest that $\mathbf{3}$ is formed from $\mathbf{2}$ by the action of sulfuric acid.

\section{Reaction Scheme}

The reaction of diphenylethylene with sulfuric acid is intrinsically more complex than that with $\mathrm{CF}_{3} \mathrm{SO}_{3} \mathrm{H}$. The rapid-scan spectrum obtained immediately after the mixing of diphenylethylene and sulfuric acid usually gives two absorption maxima. The one at $435 \mathrm{~nm}$ may certainly be assigned to the monomer cation derived from simple protonation. This process was studied in detail in the previous publication ${ }^{1}$ with $\mathrm{CF}_{3} \mathrm{SO}_{3} \mathrm{H}$ as initiator. The assignment of the 465-nm species is less unambiguous. This species is formed when the concentration of sulfuric acid is $100 \%$ or more, and is more short-lived. These sulfuric acids should contain the $\mathrm{SO}_{3}$ species. In fact, the 465-nm peak appeared when gaseous $\mathrm{SO}_{3}$ was bubbled into a dichloroethane solution of DPE in a separate experiment. ${ }^{8}$ Under the reaction conditions where only the 465 -nm peak was observed in the rapid-scan spectrum, the major quenching products were the $1: 1$ adduct of diphenylethylene and $\mathrm{H}_{2} \mathrm{SO}_{4}$ or its elimination product ( 2 and $\mathbf{3}$ ).

Some years ago, Fleischfresser et al. studied the electron transfer from diphenylethylene to $\mathrm{SbCl}_{5}$ and found the formation of an unstable intermediate absorbing at $465 \mathrm{~nm} .{ }^{6}$ They assumed this species to be a charge-transfer complex of diphenylethylene and : $b \mathrm{bl}_{5}$. Hayashi and coworkers recently studied primary ionic species of diphenylethylene produced by high-energy radiation and concluded that monomeric radical cation 4 absorb at 395 and $550 \mathrm{~nm}^{7}$ These results suggests that the $465-\mathrm{nm}$ species formed from diphenylethylene and $\mathrm{SO}_{3}$ is a chargetransfer complex.<smiles></smiles>

The reaction of diphenylethylene and sulfuric acid may now be summarized as in Scheme I.

The course of the formation of the cyclic sulfones 
Table I. Quenching experiment

\begin{tabular}{|c|c|c|c|c|c|c|c|}
\hline \multirow{2}{*}{ No. } & \multirow{2}{*}{$\frac{\mathrm{DPE}}{\mathrm{mM}}$} & \multirow{2}{*}{$\frac{\mathrm{H}_{2} \mathrm{SO}_{4}^{\mathrm{a}}}{\mathrm{mM}}$} & \multirow{2}{*}[\mathrm{H}_{2}\mathrm{SO}_{4}]{$/[\mathrm{DPE}]$} & \multirow{2}{*}{ Reaction time } & \multirow{2}{*}{$\frac{\text { Coversion }^{\mathrm{b}}}{\%}$} & \multicolumn{2}{|c|}{$\begin{array}{l}\text { Approximate product } \\
\text { distribution } 1 \%\end{array}$} \\
\hline & & & & & & 2 & 3 \\
\hline 1 & 0.45 & 21 & 47 & $0.05 \mathrm{~s}$ & $20-30$ & 100 & 0 \\
\hline 2 & 0.58 & 23 & 40 & $20 \mathrm{~min}$ & 100 & 63 & 37 \\
\hline 3 & 0.58 & 27 & 47 & $60 \mathrm{~min}$ & 100 & 50 & 50 \\
\hline 4 & 8.7 & 13 & 1.5 & $15 \mathrm{~min}$ & $80-90$ & 100 & 0 \\
\hline 5 & 37 & 56 & 1.5 & $90 \mathrm{~min}$ & $80-90$ & 100 & 0 \\
\hline 6 & 4.8 & 27 & 5.6 & $16 \mathrm{~min}$ & 100 & 92 & 8 \\
\hline
\end{tabular}

a $106 \%$ Sulfuric acid.

b Determined from liquid chromatograms of the quenched product.

c Determined by the relative height of peaks at $8 \mathrm{~min} 30 \mathrm{~s} \mathrm{(2)}$ and at $9 \mathrm{~min}(3)$ in liquid chromatograms. The extinction coefficient of these compounds at $254 \mathrm{~nm}$ was assumed to be close.

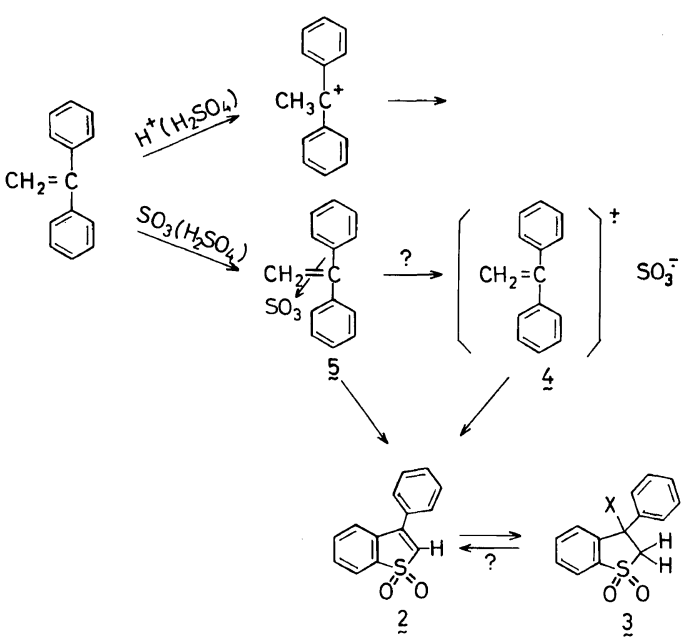

Scheme I

from charge-transfer complex $\mathbf{5}$ is not confirmed. In the reaction of diphenylethylene and $\mathrm{SbCl}_{5},{ }^{6}$ the initially-formed charge-transfer complex is supposedly transformed into a radical pair of $\mathrm{DPE}^{+}$and $\mathrm{SbCl}_{5}{ }^{-}$which eventually gives the dimer dication of diphenylethylene. Sulfone $\mathbf{2}$ may thus be formed directly from $\mathbf{5}$ or via radical pair $\mathbf{4}$. The latter pathway remains a possibility, though the radical cation was not observed spectrally. Sulfone 3 may be formed by the addition of $\mathrm{H}_{2} \mathrm{O}$ or $\mathrm{H}_{2} \mathrm{SO}_{4}$ to 2 .

An alternative mechanism for the sulfone formation involves the insertion of $\mathrm{SO}_{3}$ onto the methylene $\mathrm{C}-\mathrm{H}$ bond, followed by dehydration and cyclization.* The cyclization process may in fact be the same in both schemes. The observation of the charge-transfer band at $465 \mathrm{~nm}$ indicates a strong interaction of diphenylethylene and $\mathrm{SO}_{3}$, even in Scheme II.

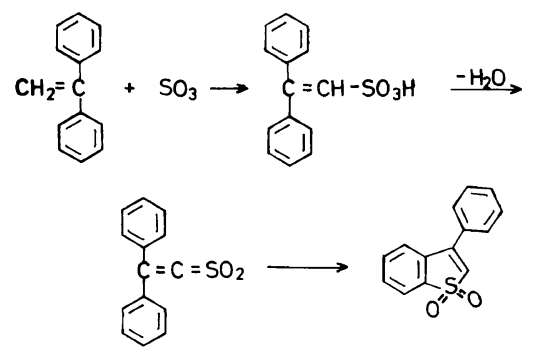

Scheme II

\section{REFERENCES}

1. K. Takarabe and T. Kunitake, Polym. J., 12, 239 (1980).

2. M. Masure, G. Sauvet, and P. Sigwalt, J. Polym. Sci., Polym. Chem. Ed., 16, 3065 (1978).

3. K. Ikeda, T. Higashimura, and S. Okamura, Kobunshi Kagaku, 26, 364 (1969).

4. T. Kunitake and K. Takarabe, Polym. J., 10, 105 (1978).

* This mechanism was suggested to us by Prof. A. Senning of University of Aarhus during his visit to Kyushu University. 
5. C. J. Pouchert and J. R. Campbell, Ed., "The Aldrich Library of NMR Spectra," Vol. X, Aldrich Chem. Co., Milwaukee, 1974, $20 \mathrm{C}$.

6. B. E. Fleischfresser, W. J. Cheng, J. M. Pearson, and
M. Szwarc, J. Am. Chem. Soc., 90, 2172 (1968).

7. K. Hayashi, M. Irie, D. Lindenau, and W. Schnabel, Radiat. Phys. Chem., 11, 139 (1978).

8. K. Takarabe, unpublished results. 